This is a postprint version of the following published document:

Garcia Armada, A. ; Hanzo, L. (2015). A Non-Coherent Multi-User Large Scale SIMO System Relaying on M-ary DPSK. Communications (ICC), 2015 IEEE International Conference on, pp.2517 - 2522. Available in http://dx.doi.org/10.1109/ICC.2015.7248703

(C) 2015. IEEE. Personal use of this material is permitted. Permission from IEEE must be obtained for all other uses, in any current or future media, including reprinting/republishing this material for advertising or promotional purposes, creating new collective works, for resale or redistribution to servers or lists, or reuse of any copyrighted component of this work in other works. 


\title{
A Non-Coherent Multi-User Large Scale SIMO System Relaying on M-ary DPSK
}

\author{
Ana Garcia Armada ${ }^{+}$and Lajos Hanzo* \\ + University Carlos III of Madrid, Department of Signal Theory and Communications \\ e-mail: agarciaetsc.uc3m.es \\ * School of ECS, University of Southampton, SO17 1BJ, United Kingdom. \\ e-mail: Ih@ecs.soton.ac.uk
}

\begin{abstract}
A non-coherent detection assisted Differential Phase Shift Keying aided large-scale MIMO system is designed in a wireless uplink where multiple single-antenna users are transmitting to the base station's receiver equipped with a very large number of receive antennas. We show that the signal to interference plus noise ratio (SINR) scales with the number of receive antennas, which confirms the same scaling law found in coherent systems. We propose a range of constellation designs that allow us to separate the users' signals at the receiver by relying only on the knowledge of the average received power per user. We analyse the error probability and provide insights into the beneficial selection of the constellation parameters. Finally, we provide some numerical results showing that our proposals require a lower number of receive antennas to achieve a given error probability than other non-coherent benchmark schemes available in the literature, while they are not far from an equivalent coherent system relying on realistic channel estimation settings.
\end{abstract}

\section{INTRODUCTION}

Large scale multiple input-multiple output (LS-MIMO) systems have attracted substantial interest in the research community because of their potential to provide unprecedented gains in terms of their spectral- and energy-efficiency [1],[2]. Indeed, by increasing the number of antennas beyond those of the operational standards, both the data rate and the integrity can be improved impressively while the required transmit power is reduced. Moreover, this performance can be achieved with the aid of low-complexity linear processing [3]. One of the main bottlenecks of LS-MIMO is the need to obtain the Channel State Information (CSI) of numerous channels for coherent detection or for Transmit Pre-Coding (TPC). In order for the channel estimation to be feasible, LS-MIMO systems tend to assume Time Division Duplex (TDD) operation, where the pilot sequences are transmitted in the uplink (UL) for estimating the UL channel and the downlink (DL) channel is then assumed to be identical during the TPC transmission. Furthermore, the pilot contamination due to the non-orthogonallity of the pilot sequences used in neighbor cells seriously compromises the performance of these systems [4]. Even though there is some ongoing research on ameliorating this problem [5], there are still open challenges for further research.

As a design alternative, in this treatise we focus on noncoherent detection as a means to circumventing channel estimation and additionally mitigating the impact of other impairments, such as phase noise. For MIMO systems, the authors of [6] proposed unitary space-time modulation for avoiding the need for CSI estimation at the receiver. This scheme was shown to work well for long coherence time intervals or for high Signal to Noise Ratios (SNR). Here we focus our attention on the energy-efficiency potential of increasing the number of antennas and we will seek solutions that work at low SNRs. In this realm we can cite the contribution of [7], where an Amplitude Shift Keying (ASK) system was conceived. Manolakos et al. [7] show that in terms of the scaling law of achievable rates, the performance of the proposed non-coherent system is no different from that achievable under the idealised simplifying assumption of having perfect CSI at the transmitters and the receiver. However, the design proposed in [7] requires an excessive number of antennas for a reasonable performance. As an attractive design alternative, a Differential Quaternary Phase Shift Keying (DQPSK) system is presented in [8], relying on a particular channel model that makes the MIMO system resemble an Impulse Radio-Ultra Wide Band (IR-UWB) system. Under this particular channel model the users can be spatially separated based on their nonoverlapping 'power-space' profiles, but this channel model cannot be exploited in general.

Against this background our novel contribution is that we propose several constellations based on Differential $M$-ary Phase Shift Keying (DMPSK) that allow us to use differential detection and separate the signals of multiple users merely relying on the knowledge of their received signal powers whilst leveraging the advantages of using an increased number of receive antennas. We will show that our proposal performs better than the benchmarker of [7] and is not far from the performance of an equivalent coherent system operating under realistic channel estimation assumptions. 


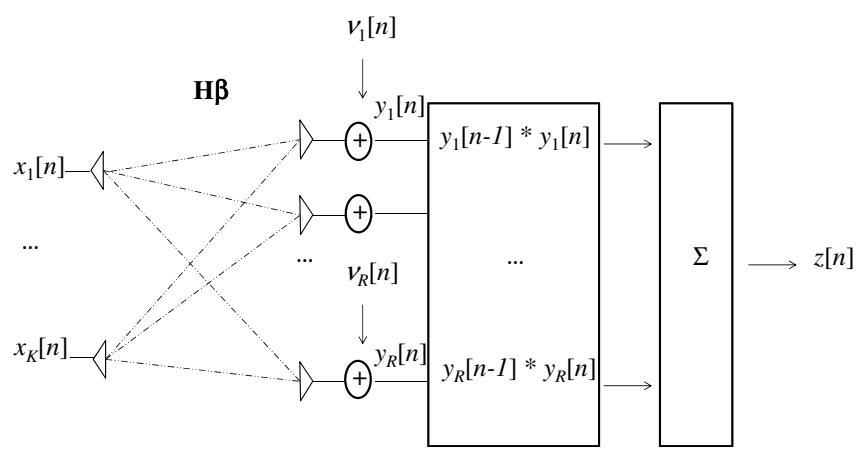

Fig. 1. System block diagram.

\section{SySTEM MODEL}

We consider a multi-user single input-multiple output (SIMO) uplink scenario, where a single base station (BS) is equipped with $R$ receive antennas (RA) to receive the signals transmitted from $K$ Mobile Stations (MSs), or users. Figure 1 shows the block diagram of the complete system.

We denote the signal to be transmitted by user $j$ at time instant $n$ as $x_{j}[n]$ and the signals from all users are grouped in the $(K \times 1)$-element vector $\boldsymbol{x}$. Each of the signals $x_{j}[n]$ arriving differentially encoded from $s_{j}[n]$ as

$$
x_{j}[n]=x_{j}[n-1] s_{j}[n], n>1 .
$$

The symbols $s_{j}[n]$ are independent and belong to an $M$ ary constellation $\mathfrak{M}_{j}=\left\{s_{j, m}, m=0,1, \ldots, M-1\right\}$, which may in fact be different for each user, as we demonstrate later, where $\left|s_{j, m}[n]\right|=1$. Each $x_{j}[0]$ is a known reference symbol taken from the same constellation. The propagation channel is represented by the $(R \times K)$-element channel matrix $\boldsymbol{H}$ with the components $h_{i, j}$ modeling the propagation from user $j$ to the $i$-th antenna of the BS. The coefficients $h_{i, j}$ are circularly symmetric complex Gaussian random variables $h_{i, j} \sim C N(0,1)$, representing Rayleigh fading. The effects of large-scale fading are taken into account with an additional power term. Explicitly, the signals of the different users may be received at a different average power, either because they are allowed to use a different transmit power or because their path loss is not compensated by power control. Without loss of generality we will assume that the signal of user 1 is received with unit power and the others have a relative gain of $\beta_{j}$ with respect to user 1. The vector $\boldsymbol{y}$ groups the signal received at each of the BS antennas at each time instant $y_{i}[n]$. Then the signal received at the BS is formulated as

$$
\boldsymbol{y}=\boldsymbol{H} \beta \boldsymbol{x}+\boldsymbol{v},
$$

where the dependence on $n$ is dropped for ease of notation. Here $\boldsymbol{v}$ is the $(R \times 1)$-element vector of AWGN components $v_{i}[n] \sim C N\left(0, \sigma^{2}\right)$ and the diagonal matrix $\boldsymbol{\beta}=\operatorname{diag}\left\{\sqrt{\beta_{j}}\right\}$ contains the power terms associated with the transmissions of the different users $j=1, \ldots, K, \beta_{1}=1, \beta_{j} \geq 0, j \neq 1$. We define the reference SNR as

$$
\rho=\frac{\sum_{j=1}^{K} \beta_{j}}{\sigma^{2}} .
$$

At the receiver, the phase difference of two consecutive symbols received at each antenna is non-coherently detected, assuming that the channel stays time-invariant for these two symbols, $h_{i j}[n-1]=h_{i j}[n]=h_{i j}, i=1, \ldots, R, j=1, \ldots, K$, and they are all added to give the decision variable of

$$
z[n]=\frac{1}{R} \sum_{i=1}^{R} y_{i}[n-1]^{*} y_{i}[n],
$$

that contains information and interference gleaned from all users

$$
\begin{aligned}
& z[n]=\frac{1}{R} \sum_{j=1}^{K} \sum_{i=1}^{R}\left|h_{i j}\right|^{2} \beta_{j} s_{j}[n] \\
& +\frac{1}{R} \sum_{\substack{j=1 \\
k}}^{K} \sum_{\substack{k=1 \\
k \neq j}}^{K} \sum_{i=1}^{R} h_{i j}^{*} h_{i k} x_{j}^{*}[n-1] \sqrt{\beta_{j} \beta_{k}} x_{k}[n] \\
& +\frac{1}{R} \sum_{i=1}^{R} v_{i}^{*}[n-1] \sum_{j=1}^{K} h_{i j} \sqrt{\beta_{j}} x_{j}[n] \\
& +\frac{1}{R} \sum_{i=1}^{R} v_{i}[n] \sum_{j=1}^{K} h_{i j}^{*} \sqrt{\beta_{j}} x_{j}^{*}[n-1]+\frac{1}{R} \sum_{i=1}^{R} v_{i}^{*}[n-1] v_{i}[n] .
\end{aligned}
$$

From the Law of Large Numbers we know that

$$
\frac{1}{R} \sum_{i=1}^{R}\left|h_{i j}\right|^{2} \stackrel{R \rightarrow \infty}{=} 1
$$

almost surely. Then, if we define the joint received symbol as

$$
\varsigma[n]=\sum_{j=1}^{K} \beta_{j} s_{j}[n]
$$

we have

$$
z[n] \stackrel{M \rightarrow \infty}{=} \varsigma[n]+\text { noise terms }
$$

and we can obtain an estimate of $\varsigma[n]$ from $z[n]$ as

$$
\hat{\zeta}[n]=\arg \min \{|\hat{\zeta}[n]-z[n]|, \hat{\zeta}[n] \in \mathfrak{M}\},
$$

where the joint constellation $\mathfrak{M}=\left\{\varsigma_{m}, m=0,1, \ldots, \mathfrak{K}-1\right\}$ of cardinality $\mathfrak{K}=M^{K}$ is obtained from the superimposed combinations of the constellation points of $\mathfrak{M}_{j}$ as $\left\{\beta_{1} s_{1, m^{(1)}}+\right.$ $\left.\beta_{2} s_{2, m^{(2)}}+\ldots+\beta_{K} s_{K, m^{(k)}}, m^{(j)}=0,1, \ldots, M-1\right\}$. In the next section we will show that as $R$ grows bigger this lowcomplexity estimate offers a good performance. In Section IV the signal constellations $\mathfrak{M}_{j}$ will be specifically designed for efficiently recovering the users data $s_{j}[n]$ from the jointly detected symbol $\hat{\varsigma}[n]$. 


$$
\begin{aligned}
& i[n]=\zeta[n]-z[n]=\underbrace{\sum_{j=1}^{K}\left[\beta_{j} s_{j}[n]\left(1-\frac{1}{R} \sum_{i=1}^{R}\left|h_{i j}\right|^{2}\right)\right]-\frac{1}{R} \sum_{\substack{j=1 \\
k \neq j}}^{K} \sum_{\substack{k=1 \\
k \neq j}}^{K} h_{i j}^{*} h_{i k} x_{j}^{*}[n-1] \sqrt{\beta_{j} \beta_{k}} x_{k}[n]}_{i_{1}[n]} \\
& -\underbrace{\left(\frac{1}{R} \sum_{i=1}^{R} v_{i}^{*}[n-1] \sum_{j=1}^{K} h_{i, j} \sqrt{\beta_{j}} x_{j}[n]+\frac{1}{R} \sum_{i=1}^{R} v_{i}^{[n]} \sum_{j=1}^{K} h_{i, j}^{*} \sqrt{\beta_{j}} x_{j}^{*}[n-1]\right.}_{i_{2}[n]} \underbrace{\left.\frac{1}{R} \sum_{i=1}^{R} v_{i}^{*}[n-1] v_{i}[n]\right)}_{i_{3}[n]}
\end{aligned}
$$

\section{AnAlysis of the Signal to Interference Plus NOISE RATIO}

The Signal to Interference plus Noise Ratio (SINR) is defined as the ratio of the signal power to the power of AWGN noise plus interference created by the detection process. When detecting $\hat{\zeta}[n]$ from $z[n]$, the interference plus noise arises from the noise terms in (8) and from equality in (6) not being met due to a finite value of $R$. Hence the interference plus noise term $i[n]$ is shown in (10) in the next page. By exploiting the properties of Gaussian and Wishart matrices (omitted here for space economy), the expectation of the power of the different terms of $i[n]$ in (10), $I=E\left\{\left|i[n]^{2}\right|\right\}$ can be shown to be

$$
\begin{gathered}
I_{1}=\frac{1}{R}\left(\sum_{j=1}^{K} \beta_{j}\right)^{2} \\
I_{2}=\frac{2}{R} \sigma^{2} \sum_{j=1}^{K} \beta_{j} \\
I_{3}=\frac{1}{R} \sigma^{4} .
\end{gathered}
$$

Then the SINR obeys

$$
\operatorname{SINR}=\frac{E\left\{|\zeta|^{2}\right\}}{I}=\frac{R \sum_{j=1}^{K} \beta_{j}^{2}}{\left(\sum_{j=1}^{K} \beta_{j}\right)^{2}+2 \sigma^{2} \sum_{j=1}^{K} \beta_{j}+\sigma^{4}} .
$$

For a high SNR, given by $\rho$ (3), only $I_{1}$ is significant, so that $\operatorname{SINR}_{H}=R \frac{\sum_{j=1}^{K} \beta_{j}{ }^{2}}{\left(\sum_{j=1}^{K} \beta_{j}\right)^{2}}$, while for a low $\rho$ the main dominant term is $I_{3}$ so that $\operatorname{SINR}_{L}=R \frac{\sum_{j=1}^{K} \beta_{j}^{2}}{\sigma^{4}}$. We can see that upon increasing the number of antennas at the BS the SINR increases proportionately. Hence, the energy-efficiency with $R$, obeying the same scaling law as for coherent systems associated with perfect CSI [2].

\section{DESIGN OF THE INPUT SIGNAL CONSTELlationS}

In order to separate the users' signals at the BS, the constellations $\mathfrak{M}_{j}$ must be specifically designed so that their symbols can still be uniquely and unambiguously distinguished upon superimposing the transmitted signals from all users. More explicitly, the superimposed transmitted constellation $\mathfrak{M}$ obtained from all legitimate combinations of the constellation points of $\mathfrak{M}_{j}$ should have $M^{K}$ uniquely distinguishable points.
Provided this is accomplished, the individual users' data symbols $s_{j}[n]$ can be directly obtained from the detected joint symbols $\hat{\zeta}[n]$ by the low-complexity demapping operation of

$$
\left\{\hat{s}_{j}[n]\right\}=\arg \min \left\{\left|\sum_{j=1}^{K} \beta_{j} \hat{s}_{j}[n]-z[n]\right|, \hat{s}_{j}[n] \in \mathfrak{M}_{j}\right\} .
$$

By considering the minimum distance of the constellation

$$
d_{\text {min }}=\min \left\{\left|\varsigma_{m}-\varsigma_{m^{\prime}}\right|, 1 \leq m \leq \mathfrak{K}, 1 \leq m^{\prime} \leq \mathfrak{K}, m^{\prime} \neq m\right\},
$$

we can define its normalized minimum distance (NMD) as

$$
\overline{d_{\min }}=\frac{d_{\min }}{\sqrt{\sum_{j=1}^{K} \beta_{j}^{2}}} .
$$

We will see in Section V that as expected, this NMD determines the attainable error performance. Here we propose and analyze two possible constellation designs.

\section{A. Constellation design A}

The $K$ users transmit their symbols according to the following $M$-DPSK constellations:

$$
\mathfrak{M}_{j}^{A}=\left\{\frac{2 \pi m}{M} L^{1-j}, m=0,1, \ldots, M-1\right\}, j=1, \ldots, K, L \geq M .
$$

An example of the users' and joint costellations is shown in Figure 2 for $K=2$.

\section{B. Constellation design $B$}

All the users transmit symbols of the same standard $M$ DPSK constellation.

$$
\mathfrak{M}_{j}^{B}=\left\{\frac{2 \pi m}{M}, m=0,1, \ldots, M-1\right\}, j=1, \ldots, K .
$$

Their signals require different powers $\beta_{j}$ to be separated. An example of the users' and joint costellations is shown in Figure 3 for $K=2$.

\section{Minimum distance}

The minimum distance of the joint constellation (16) depends on the design parameters, namely $L$ and $\beta_{j}$ for design $\mathrm{A}$ and solely on $\beta_{j}$ for design $\mathrm{B}$. The specific values of the design parameters that achieve the maximum NMD (17) will be shown in Section $\mathrm{V}$ to provide the best error performance. These values can be found by exhaustive search, since the 


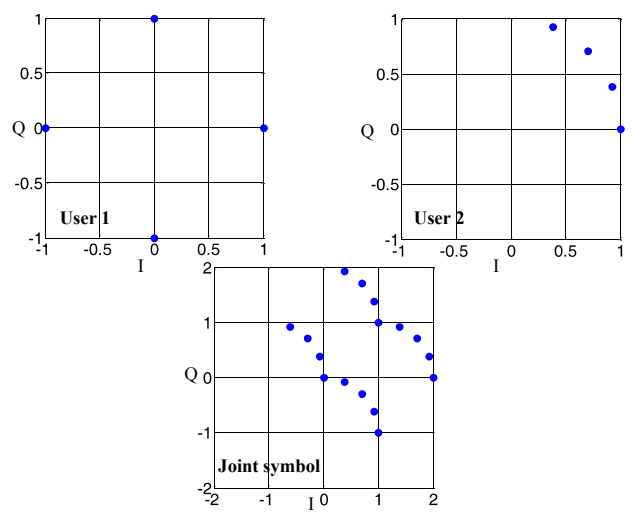

Fig. 2. Constellation A for $K=2$ users, $M=L=4$ and $\beta_{2}=1$.

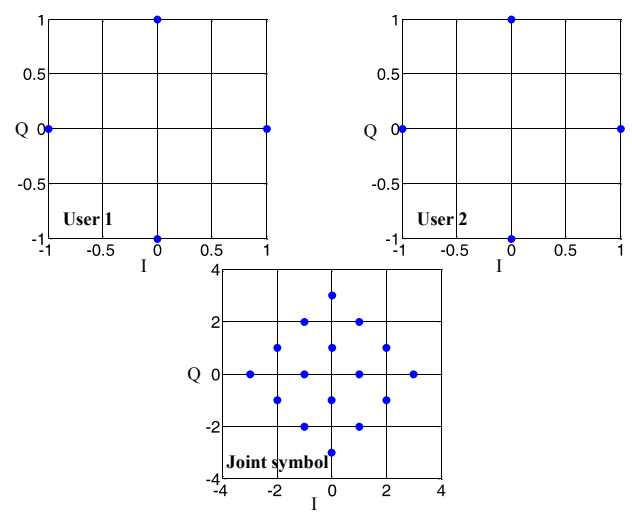

Fig. 3. Constellation B for $K=2$ users, $M=4$ and $\beta_{2}=2$.

number of combinations is computationally manageable. The geometry of the constellations facilitates the following analysis.

For constellation design $\mathrm{A}$, which relies on $L=M$ and $\beta_{j}=1$ we can see that the minimum distance is given by the constellation of user $K$, namely by

$$
d_{\text {min }}^{A}=\left|1-e^{\frac{j 2 \pi}{M^{2}}}\right| \simeq \sin \left(\frac{2 \pi}{M^{K}}\right) \simeq \frac{2 \pi}{M^{K}}
$$

and the NMD is

$$
\overline{d_{\min }^{A}} \simeq \frac{2 \pi}{M^{K} \sqrt{K}},
$$

whose value decreases exponentially, as $K$ is increased.

For constellation design $\mathrm{B}$, the minimum distance of the constellation for $K=1$ user is given by the unit-energy DPSK constellations, that is, $d_{\min }^{(1)}=2$ for $M=2, d_{\min }^{(2)}=\sqrt{2}$ for $M=4$, $d_{\min }^{(3)}=2-\sqrt{2}$ for $M=8$. For $M=2$ and $M=4$, supporting additional users creates new joint constellations where this $d_{\text {min }}$ can be preserved, provided that $\beta_{j} \geq 2^{j-1}$. In this case, the $K$-user constellation contains separate replicas of the smaller constellations obtained for $(K-1)$ users, with a separation of at least $d_{m i n}$. This implies that the NMD decreases as $\beta_{j}$ is increased, so that the maximum normalized $d_{\min }$ is achieved for $\beta_{j}=2^{j-1}$, which has a value of

$$
\overline{d_{\text {min }}^{B}}=\frac{d_{\min }^{(l)}}{\sqrt{\sum_{j=1}^{K} 2^{2(j-1)}}}=\frac{\sqrt{3} d_{\min }^{(l)}}{\sqrt{4^{K}-1}}, l=1,2 .
$$

From this equation we can observe a less substantial reduction of the maximum $\overline{d_{\min }}$, when $K$ is increased for design B. For $M>4$ the geometry becomes more complicated and the values of $\beta_{j}$ that maximize $\overline{d_{\min }}$ can be found by exhaustive search. However, they do not in general obey $\beta_{j}=2^{j-1}$. For example, for $M=8$ and $K=2$ users, the maximum $\overline{d_{\min }}$ is found to be for $\beta_{2}=1.765$.

\section{ERROR PROBABILITY ANALYSIS}

The error probability of the joint symbols $\varsigma[n]$ may be found using the Union Bound and assuming that the interference plus noise $i[n]$ in (10) is Gaussian, which is justified by the Central Limit Theorem. Let us first define the pairwise error probability as the probability of detecting symbol $\mathrm{m}^{\prime}$, when symbol $m$ was transmitted, that is [9]

$$
P e_{m m^{\prime}}=Q\left(\sqrt{\frac{d_{m m^{\prime}}^{2}}{2 I}}\right), m, m^{\prime} \in\{0,1, \ldots \mathfrak{K}-1\}, m^{\prime} \neq m,
$$

where the distance between points $m$ and $m^{\prime}$ of the joint constellation is $d_{m m^{\prime}}=\left|\varsigma_{m}-\varsigma_{m^{\prime}}\right|$. Then we can upper-bound the symbol error probability (SEP) $P_{e}$ as

$$
P_{e} \leq \frac{1}{\mathfrak{K}} \sum_{\substack { m=0 \\
\begin{subarray}{c}{0 \leq m^{\prime} \leq \mathfrak{K}-1 \\
m^{\prime} \neq m{ m = 0 \\
\begin{subarray} { c } { 0 \leq m ^ { \prime } \leq \mathfrak { K } - 1 \\
m ^ { \prime } \neq m } }\end{subarray}}^{\mathfrak{K}-1} Q\left(\sqrt{\frac{d_{m m^{\prime}}^{2}}{2 I}}\right) .
$$

If we consider the minimum constellation distance $d_{\min }(16)$, we can further upper-bound the SEP as

$$
P_{e} \leq(\mathfrak{K}-1) Q\left(\sqrt{\frac{d_{\min }^{2}}{2 I}}\right) .
$$

We can also find a lower bound to the SEP as follows. If we denote the minimum distance of each constellation point $m$ from its nearest neighbor by $d_{m i n}^{m}$, the SEP satisfies [9]

$$
P e \geq \frac{1}{\mathfrak{K}} \sum_{m=0}^{\mathfrak{K}-1} Q\left(\frac{d_{\min }^{m}}{\sqrt{2 I}}\right) .
$$

The power of the noise plus interference $I$ was found in Section III to be

$$
I=\frac{\left(\sum_{j=1}^{K} \beta_{j}\right)^{2}+2 \sigma^{2} \sum_{j=1}^{K} \beta_{j}+\sigma^{4}}{M} .
$$

Recalling that we have $I=\frac{\sum_{j=1}^{K} \beta_{j}^{2}}{\operatorname{SINR}}(14)$, we can also express (25) as

$$
P e \leq(\mathfrak{K}-1) Q\left(\sqrt{\frac{d_{\min }^{2}}{2 \sum_{j=1}^{K} \beta_{j}^{2}} \operatorname{SINR}}\right) .
$$


This implies that for a given SINR, the error performance depends on the normalized $d_{\min }$ (17). Then, according to the discussion in Section IV, constellation B will have a better performance than constellation $\mathrm{A}$. We can find the constellation parameters that optimize the error probability by maximizing the normalized $d_{\min }$.

The same approximations also apply to the individual SEP of each of the users, provided that the distances between the constellation points that encode the same user's symbol are not evaluated.

\section{PERformance EVAluation}

In this section block fading is assumed, where the channels are kept constant during the transmission of a long symbol burst and they vary randomly between bursts. Figure 4 shows the SEP performance of the $K=2$ users DQPSK system compared to previous work [7] at $\mathrm{SNR}=0 \mathrm{~dB}$ using the same modulation order of $M=4$. The upper bound and simulated SEP $\left(P_{e}\right)$ of [7] is compared to the $P_{e}$ evaluated by simulation and the bounds for both of the proposed constellation designs. For design A we have used $L=4$ and $\beta_{2}=1$, while for design B $\beta_{2}=2$. We can see that the proposed designs achieve a better SEP performance. Moreover, they require a lower number of RAs at the BS to obtain an adequate SEP, in particular for constellation design $\mathrm{B}$.

Figure 5 shows the performance of constellation B for DBPSK, DQPSK and 8-DPSK modulations with maximum NMD, where the accuracy of the SEP bounds can be contrasted to the simulation results. We can see that the upper bound is tight. The lower bound, although looser, provides also a good approximation to the SEP. For higher SINR values both bounds become quite accurate.

For constellation design B with DQPSK the values that achieve the best SEP for a given $\rho$ are $\beta_{j}=2^{j-1}$. For this case, Figure 6 shows the SEP performance given by the upper and lower bounds as the number of users $K$ is increased. We can see that up to $K=4$ simultaneous users can be supported by the same time and frequency resource at an SNR as low as $0 \mathrm{~dB}$. The SEP performance can be further improved with the aid of appropriate coding schemes that are the subject of our future work.

We also compare the performance of $K=2$ users and DQPSK to that achieved by a coherent Maximum Ratio Combining (MRC) receiver which is known to perform well for massive MIMOs at low SNRs [3]. For this comparison we assume that the CSI is estimated with a realistic error, which is also assumed to be Gaussian. Figure 7 shows the associated performance comparison. We can see that as expected, coherent QPSK has a better performance than noncoherent DQPSK. However, for a fair comparison we should take into account the rate loss due to the insertion of pilots for channel estimation. In Long Term Evolution (LTE) this loss is $15 \%$ for a reduced number of antennas [10], while in [11] the

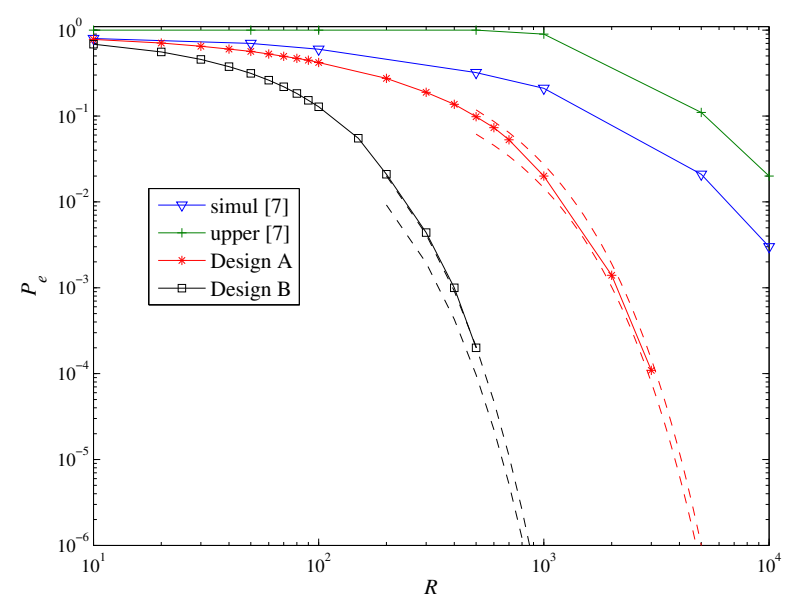

Fig. 4. Comparison of the SEP with $K=2$ users and $M=4$ at $\rho=0 \mathrm{~dB}$.

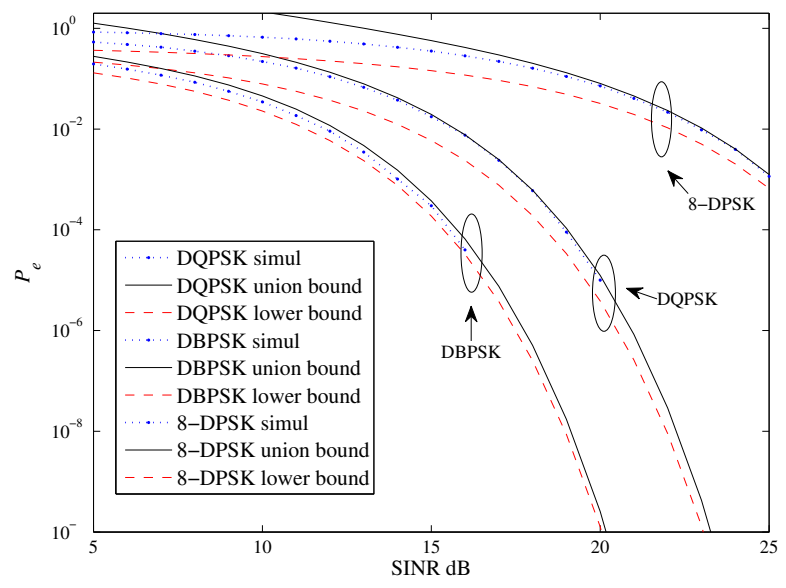

Fig. 5. SEP performance with $K=2$ users as a function of SINR for DBPSK, DQPSK and 8-DPSK modulations

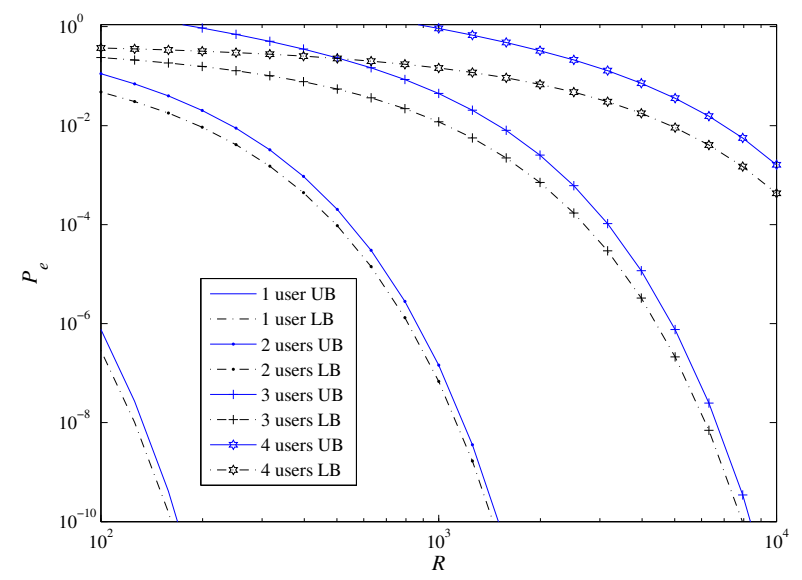

Fig. 6. SEP performance (UB: union bound, LB: lower bound) for $K=1,2,3$ and 4 users and DQPSK as a function of $R$ for $\rho=0 \mathrm{~dB}$, constellation design $B$. 


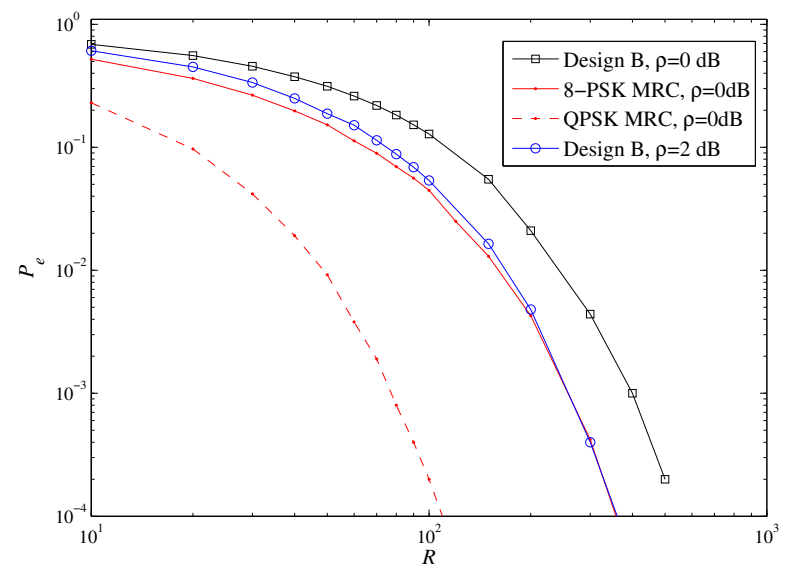

Fig. 7. SEP performance for both coherent and non-coherent detection.

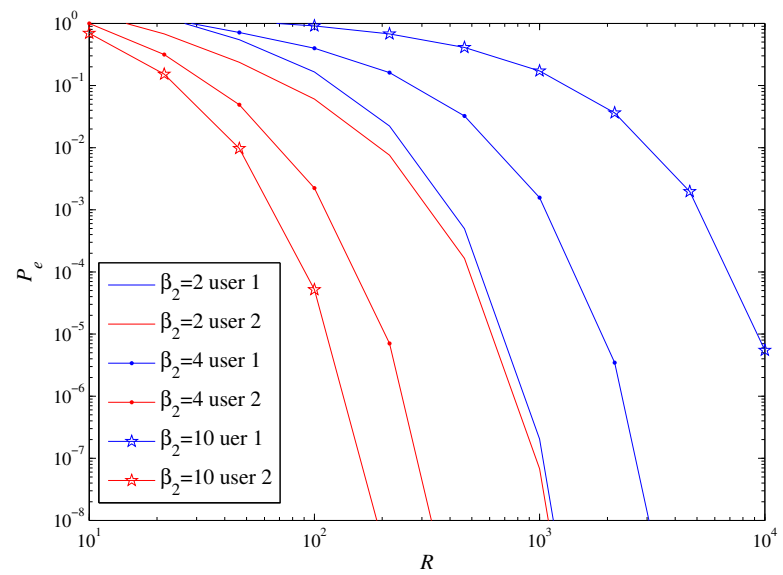

Fig. 8. SEP performance for constellation design $B$ and different values of $\beta_{2}$.

optimum amount of pilots is shown to be $35 \%-40 \%$ for low SNR with $R=16$ and an optimised number of users $K<M$. Given this background we will assume a rate-loss of $33 \%$ due to pilot overhead. This implies that for the same rate, we should compare non-coherent DQPSK to coherent 8-PSK. In that case we can see in Figure 7 that to get the same SEP, noncoherent DQPSK requires a $2 \mathrm{~dB}$ higher $\rho$. This can be deemed affordable considering its detection complexity reduction and the avoidance of the pilot contamination problems.

Finally, Figure 8 shows the performance of the individual users for $K=2$ and for constellation design $\mathrm{B}$, when the parameter $\beta_{2}$ is varied. We can see that their different received powers result in different SEP for the users. This may be of practical interest, for instance, for unequal-protection video communications.

\section{CONCLUSIONS}

We have proposed an $M$-ary DPSK system for a wireless uplink where several single-antenna aided users are trans- mitting towards the BS receiver having a large number of RAs. We have analysed the SEP versus SINR performance and proposed a pair of constellation designs that allow us to separate the users' signals at the receiver relying only on the knowledge of the average received power per user. We have provided some numerical results for verifying the accuracy of our expressions. They show that our proposals require a lower number of receive antennas to achieve a given SEP than other non-coherent schemes available in the literature, while they are not far from an equivalent throughput coherent system relying on realistic channel estimation settings. Moreover, the variation of the users' received power allows us to set up uneven error correction schemes.

\section{ACKNOWLEDGMENT}

This work was supported by projects CSD2008-00010, TEC2011-29006-C03-03 and by a mobility grant of Spanish Ministry of Education. The financial support of the UK Government's Engineering \& Physical Sciences Research Council (EPSRC) as well as that of the Research Councils UK (RCUK) and of the European Research Council's Senior Research Fellow Grant is also gratefully acknowledged.

\section{REFERENCES}

[1] E.G. Larsson, O. Edfors, F. Tufvesson, and T.L. Marzetta, "Massive MIMO for Next Generation Wireless Systems," IEEE Comm. Mag., Vol. 52, No. 2, pp. 186-195, Feb. 2014.

[2] H.Q. Ngo, E.G. Larsson, and T.L. Marzetta, "Energy and Spectral Efficiency of Very Large Multiuser MIMO Systems," IEEE Trans. Commun., Vol. 61, No. 4, pp. 1436-1449, Apr. 2013.

[3] H. Yang and T.L. Marzetta, "Performance of Conjugate and ZeroForcing Beamforming in Large-Scale Antenna Systems," IEEE Joural on Selected Areas in Commun., Vol. 31, No. 2, pp. 172-1179, Feb. 2013.

[4] T. L. Marzetta, "Noncooperative Cellular Wireless with Unlimited Numbers of Base Station Antennas," IEEE Trans. Wireless Commun., vol. 9, pp. 3590-3600, Nov. 2010.

[5] J. Zhang, B. Zhang, S. Chen, X. Mu, M. El-Hajjar, and L. Hanzo, "Pilot Contamination Elimination for Large-Scale Multiple-Antenna Aided OFDM Systems,' IEEE Journal of Selected Topics in Signal Processing, 2014, to appear.

[6] B. M. Hochwald and T. L. Marzetta, "Unitary Space-Time Modulation for Multiple-Antenna Communications in Rayleigh Flat Fading," IEEE Transactions on Information Theory, vol. 46, no. 2, pp. 543-564, 2000.

[7] A. Manolakos, M. Chowdhury, and A.J. Goldsmith, "CSI is Not Needed for Optimal Scaling in Multiuser Massive SIMO Systems," Proceedings of ISIT., Honolulu, Jul. 2014.

[8] A. Schenk and R.F.H. Fischer, "Noncoherent Detection in Massive MIMO Systems," Proc. of International ITG/IEEE Workshop on Smart Antennas., Stuttgart, Mar. 2013.

[9] J.G. Proakis, M. Salehi, Digital Communications, McGraw-Hill, NY, USA, 5th edition, 2007.

[10] 3GPP TS 36.211, "LTE; Evolved Universal Terrestrial Radio Access (EUTRA); Physical channels and modulation," version 10.0.0 Release 10, Jan. 2011.

[11] T. L. Marzetta, "How Much Training is Required for Multiuser MIMO," Proc. of IEEE Asilomar Conference on Signals, Systems and Computers, pp. 359-363, Pacific Grove, USA, Nov. 2006. 